\title{
Study on dynamics and structure of evaporation front in ethanol depending on pressure and subcooling
}

\author{
Mikhail Moiseev", and Vladimir Zhukov \\ Kutateladze Institute of Thermophysics, 630090, Novosibirsk, 1 Acad.Lavrentiev ave., Russia
}

\begin{abstract}
This paper is dedicated to study of self-sustained evaporation front in subcooled ethanol at stepwise heat generation. The data on evaporation front velocity and microstructure at different subcoolings and pressure are presented. Experiments show that development of the evaporation front drastically depends on studied parameters. At low pressures initial vapour bubbles grow without losing interface stability and initiation of evaporation fronts doesn't occur. At higher pressures the fronts propagate along the heater, and its structure depends on temperature.
\end{abstract}

\section{Introduction}

Under some conditions (nonstationary heating, low reduced pressure, etc.) onset of boiling crisis occurs via self-sustaining evaporation fronts, bypassing nucleate boiling stage. Critical heat flux in this case can be much lower than the first critical heat flux. Evaporation front is a complex nonequilibrium phenomenon which includes interrelated hydrodynamic and heat transfer processes at the interface, and also needs consideration of the arising hydrodynamic front instability, structure formation, evaporation, and thermocapillary effects. There are a few papers dedicated to model description of a self-sustained evaporation front [1-3]. But none of these models can describe front behaviour within a wide range of experimental parameters. Main flaw of most of the models is that they don't take into account the microstructure of the front, namely small-scaled hydrodynamic instability, which greatly influences heat transfer through the interface [3-5]. The lack of experimental data on evaporation front structure at various conditions also hinders its strict and complete mathematical description.

This paper describes results of experimental study on evaporation front in subcooled ethanol at stepwise heat generation and presents data on front velocity and microstructure at different subcooling and pressure.

\section{Experimental setup and procedure}

Experiments were carried out in ethanol at pressures $\mathrm{P}=0.0075 \ldots 0.101 \mathrm{MPa}$ and subcooling $\Delta \mathrm{T}_{\text {sub }}=14-43 \mathrm{~K}$. For experimental studies of heat transfer and transient

\footnotetext{
*Corresponding author: moiseevmikhail@gmail.com
} 
dynamics at non-stationary heat release the setup, shown schematically in Fig. 1, was developed.

The frame 2 with refill tank 3 and working chamber 4, housing the working section 10 , is hinged on bed 1 . The working chamber is a sealed cylindrical stainless steel vessel. The inner diameter of the chamber is $250 \mathrm{~mm}$, and the height of the workspace is $250 \mathrm{~mm}$. The working chamber is equipped with four windows 5 . The bellows 6 with an adjusting screw 7 allows creating the required pressure in the working chamber at the closed valve 8 . Heat exchanger 9 located in the bottom of the working chamber allowed setting the desired temperature of liquid.

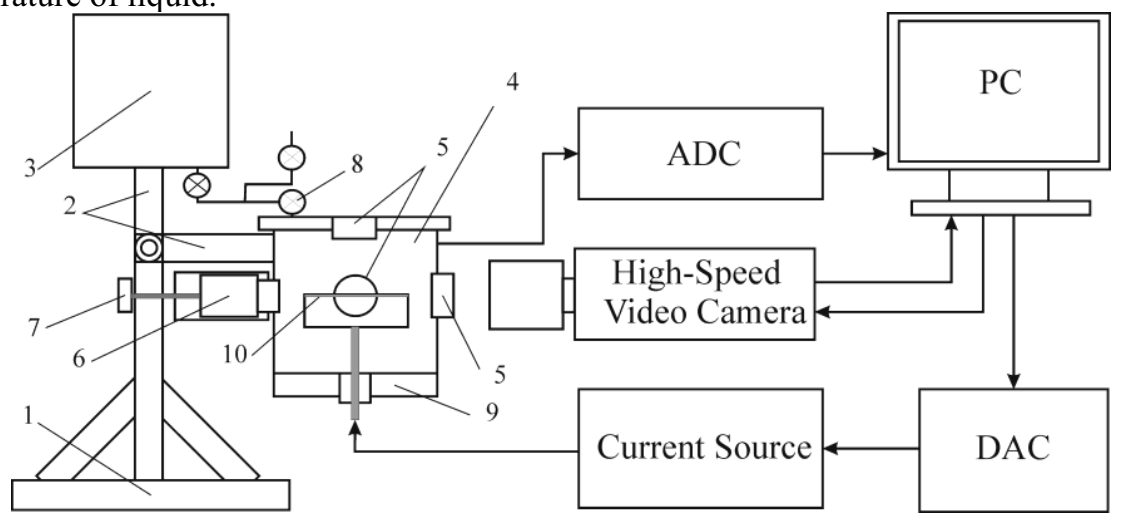

Fig. 1. Scheme of experimental setup.

The working section was made of the stainless steel tube with outer diameter of $8 \mathrm{~mm}$, wall thickness of $0.2 \mathrm{~mm}$ and length of $50 \mathrm{~mm}$. The rectangular current impulse of $420 \mathrm{~A}$ was supplied to the working section. Temperature of the heater was determined by the current impulse duration. After current was turned off, the wall temperature remained almost constant at the times of the evaporation front propagation. Numerical solution of heat equation has shown that for the time of front passage (much lesser than the time of convection development) the wall temperature before the front dropped no more than by $0.2 \mathrm{~K}$. Thus in each experiment, the propagation of self-sustained evaporation front was investigated at the pre-set constant wall superheat.

The working section temperature until the moment of vapor phase emergence was measured using temperature dependence of the heater resistance. For this purpose, two conductors of $0.05-\mathrm{mm}$ diameter were welded in the middle of the tube to measure the voltage drop in the region of $30-\mathrm{mm}$ length. In each measurement, calibration was performed by the temperature of undisturbed fluid. This technique allowed measuring the average temperature of the working section with an error less than $\pm 1.5 \mathrm{~K}$. After the vapor phase formation, the wall temperature was determined numerically based on the nonstationary heat equation. This approach is valid under conditions of our experiments since the times of the evaporation front propagation (about $50 \mathrm{~ms}$ ) were much less than the time of convection development (about $200 \mathrm{~ms}$ ).

Visual observations of vapor phase formation and propagation on the heating surface were performed using a high-speed digital video camera Phantom v7.0. The shooting rate was up to 45000 frames per second with the exposure of 19 microseconds. When analyzing the results of high-speed digital video-shooting, the line of the interfacial boundary was determined on each frame using specially developed software. The obtained data was used to study dynamic properties of evaporation front boundary. 


\section{Experimental results}

We have obtained the dependance of the front velocity on wall superheat over saturation temperature $\mathrm{T}_{\text {sat }}$ (Fig. 2). In the studied range of pressure and subcooling the front velocity is almost independent of the mentioned parameters, except the series with lowest pressures. At the lowest pressure $0.0075 \mathrm{MPa}$ initiation of evaporation fronts didn't occur. At pressure $0.026 \mathrm{MPa}$ the front velocity dependence on wall superheat becomes stronger after reaching certain temperature. This effect is due to development of the front interface instability [3-5].

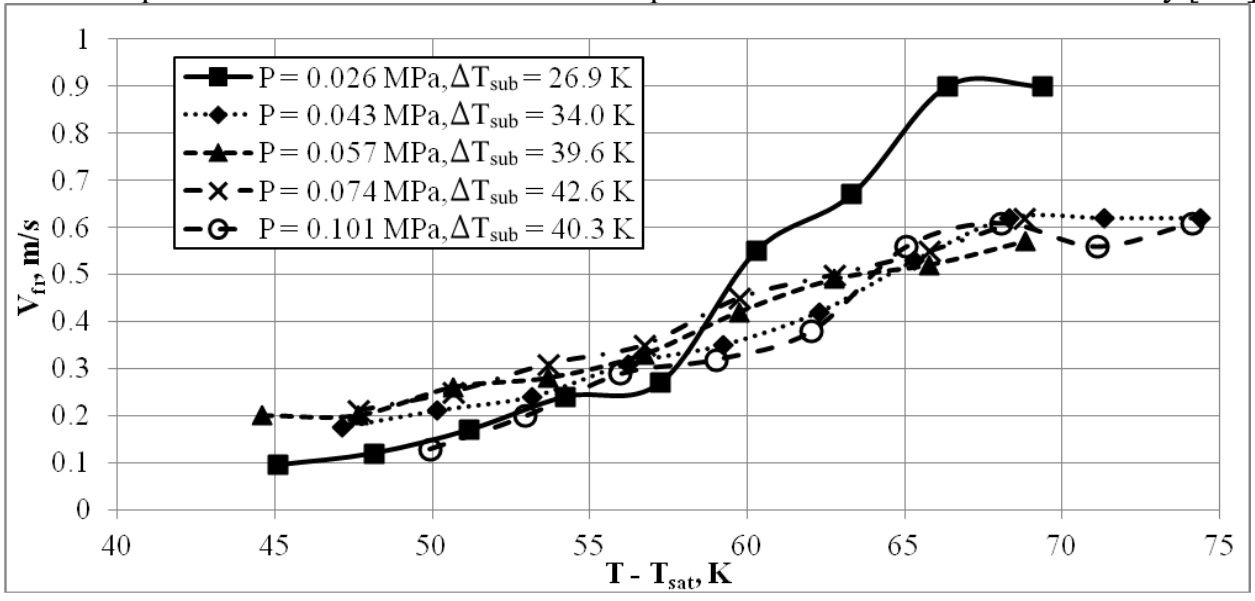

Fig. 2. Dependence of the front velocity on wall superheat at different values of pressure and subcooiling ( $\Delta \mathrm{T}$ sub).

\subsection{Mechanisms of front initiation}

The data obtained using macro videoshooting allows describing the front initiation mechanism as follows. Vapor bubble, beginning to grow at the heater surface, has smooth interface while it is within the metastable termal layer near the heater wall (Fig. 3a). At the lowest pressure $0.0075 \mathrm{MPa}$ vapor bubbles grow up to big size without losing interface stability, until they merge, and vapor layer covers the whole heater. At higher pressures, when a bubble grows out of the thermal layer into the subcooled area, condesation processes begin to compete with evaporation, and the bubble interface loses stability (Fig. $3 \mathrm{~b})$. After that evaporation front starts to propagate with its headmost point within the thermal layer (Fig. 3c). Size of the interface perturbations is comparable with the thermal layer thickness.

(a)

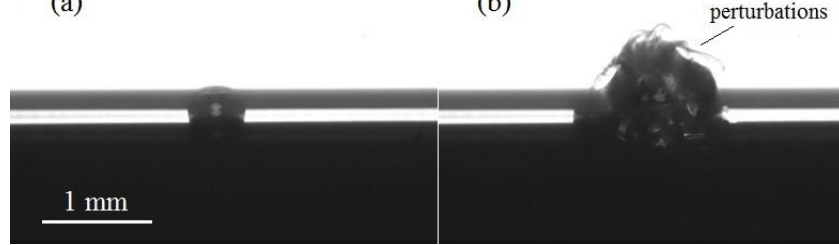

(c)

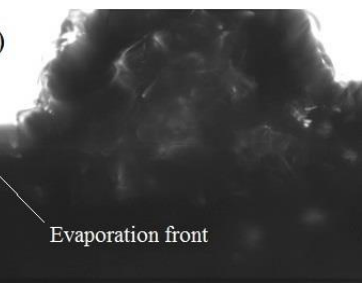

Fig 3. Evolution of a vapor bubble and emergence of the evaporation front. $P=0.101 \mathrm{MPa}$. $\Delta \mathrm{Tsub}=40.3 \mathrm{~K}$. Heating time was $40 \mathrm{~ms}(\mathrm{~T}-\mathrm{Ts} a \mathrm{t}=56 \mathrm{~K}) .(\mathrm{a})-60.15 \mathrm{~ms}$ after beginning of heat release; (b) $-62.62 \mathrm{~ms}$; (c) $-66.88 \mathrm{~ms}$.

Experiments in reflected light show that development and structure of the front significantly depend on pressure and wall superheat. As mentioned above, initiation doesn't 
occur at lowest pressure front. At higher pressure and low wall superheat the evaporation front propagating along the heater covers small caverns on the heater surface. In these caverns micro-bubbles nucleate and rapidly grow, expanding the front interface (fig. 4a). When a bubble grows out of the thermal layer, its interface loses stability, and the front moves inside the thermal layer in form of small vapor ejections until it meets new cavern, and the process repeats. Thus, the front propagates as a chain of unstable bubbles in a fractal pattern. At high wall superheat separate vapor bubbles become indistinguishable (fig. 4b). The front interface becomes quasi-flat, with only small-scaled (with wavelength $100-200 \mathrm{mcm}$ ) perturbations.
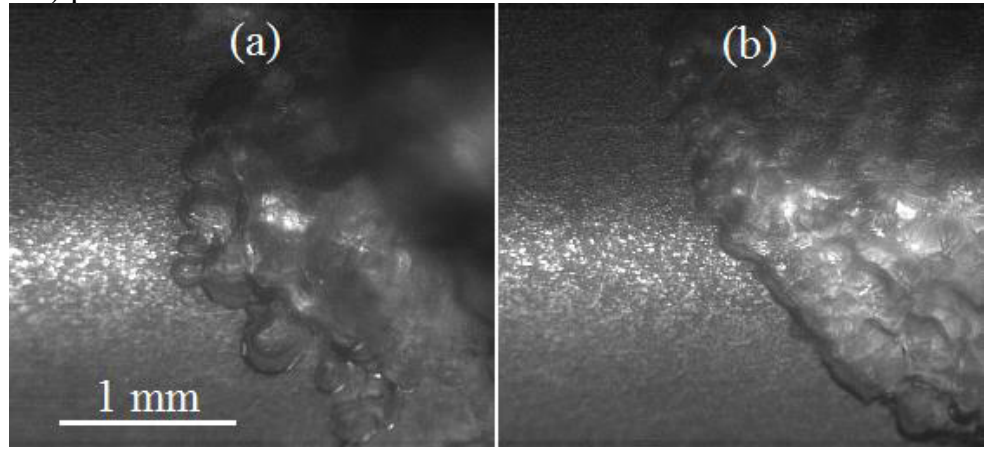

Fig 4. Evaporation front interface. $P=0.101 \mathrm{MPa} . \Delta \mathrm{Tsub}=40.3 \mathrm{~K}$. (a) $-\mathrm{T}-\mathrm{Tsat}=53 \mathrm{~K}$. (b) - T-Tsat $=68 \mathrm{~K}$.

\section{Conclusions}

Detailed study of evaporation front dynamics and microstructure in subcooled ethanol at stepwise heat generation shows the following results. Front velocity is almost independent on pressure and subcooling within the pressure range 0.043-0.101 $\mathrm{MPa}$, and has linear dependence on wall superheat. At very low pressure $(0.0075 \mathrm{MPa})$ evaporation fronts don't emerge. At moderately low pressure $(0.026 \mathrm{MPa})$ the dependence of front velocity on wall superheat becomes stronger after reaching certain critical superheat, which is corresponding to development of evaporation front instability.

Structure of the evaporation front significantly depends on wall superheat. At low superheats the front propagates as a chain of unstable bubbles in a fractal pattern. At high superheats the front interface becomes quasi-flat with small-scaled perturbations.

This research has been performed at IT SB RAS with the support of the RFBR (project No. 15-0801359)

\section{References}

1. S.P. Aktershev, V.V. Ovchinnikov, J. Eng. Thermophys. 20, 77 (2011)

2. P.A. Pavlov, V.E. Vinogradov, High Temp. 48, 683 (2010)

3. A.N. Pavlenko, V.V. Lel, Thermophys. Aeromech. 6, 251 (1999)

4. A.N. Pavlenko, E.A. Tairov, V.E. Zhukov, A.A. Levin, M.I. Moiseev, J. Eng. Thermophys. 23, 173 (2014)

5. V.E. Zhukov, A.N. Pavlenko, M.I. Moiseev, D.V. Kuznetsov, High Temp. 55, 79 (2017) 